\title{
Power system static state estimation using Kalman filter algorithm
}

\author{
Anupam Saikia* and Ram Krishna Mehta \\ Electrical Department, North Eastern Regional Institute of Science and Technology (NERIST), Itanagar, \\ Arunachal Pradesh 791109, India
}

Received 31 March 2016 / Accepted 26 October 2016

\begin{abstract}
State estimation of power system is an important tool for operation, analysis and forecasting of electric power system. In this paper, a Kalman filter algorithm is presented for static estimation of power system state variables. IEEE 14 bus system is employed to check the accuracy of this method. Newton Raphson load flow study is first carried out on our test system and a set of data from the output of load flow program is taken as measurement input. Measurement inputs are simulated by adding Gaussian noise of zero mean. The results of Kalman estimation are compared with traditional Weight Least Square (WLS) method and it is observed that Kalman filter algorithm is numerically more efficient than traditional WLS method. Estimation accuracy is also tested for presence of parametric error in the system. In addition, numerical stability of Kalman filter algorithm is tested by considering inclusion of zero mean errors in the initial estimates.
\end{abstract}

Key words: Power system state estimation, Weight least square method, Kalman filter algorithm.

\section{Introduction}

Power system state estimation is an important tool of energy management system. Power systems are monitored by supervisory control system. This kind of control system basically monitors the status of control switches, circuit breaker operation at the susbstations. These supervisory control systems also have the capability to monitor real-time system data, allowing the control centers to gather all sorts of analog measurements and circuit breaker status data from the power system [1]. The main purpose of these type of data acquisition is to maintain security of power system operation. But, the information provided by the Supervisory Control and Data Acquisition (SCADA) system may not always be true due to the presence of errors in the measurements, telemetry failures, communication noise, etc. Moreover, the collected set of measurements may not allow direct extraction of the parameter of interest. For example voltage angle at the buses cannot be measured directly. Besides that telemetering all the data of interest may require large numbers of sensors which are not feasible economically or practically. Power system state estimation processes redundant raw measurements and

*e-mail: anupam.saikia9@gmail.com available data sets in order to find an optimal estimate of the current operating states [1]. Usually bus voltage and angles are estimated in power system state estimation.

The idea of state estimation was first presented by Gauss and Legendre (around 1800). Least square state estimation in which the sum of the residual squares are minimised was the first technique which was applied for state estimation purpose [2].

State estimation was first applied to power systems by Schweppe and Wildes in the late 1960's [3]. Since then various methods have been used for power system state estimation. In paper [4], a comparative study was done between various methods. The study mainly explains the numerical stability, computational efficiency and implementation complexity of different methods.

In this paper two methods called Weight Least Square (WLS) state estimation and Kalman filter state estimation have been used for estimation of bus voltage and angles. WLS technique $[1,5]$ is a conventional technique in which weighted sum of the residual squares are minimised. Another method called Kalman filter estimation has been implemented here and it is observed that this method is more efficient computationally. In the context of complexity, this method is easier to implement. 
The theory of Kalman filter algoritm can be found in paper [6]. It was first presented around 1960. After that several applications of Kalman filters have been done in power systems. In paper [7] Kalman filer is used for distance relaying scheme and in [8] Kalman filter has been used for power system measurements for relaying. Moreover, in [9] Kalman filter has been used for harmonic analysis purpose. Since power system equations are nonlinear in nature hence extended Kalman filter [10] has been applied to Kalman filter algorithm. Power system can be modelled by different types of dynamic equations. In references $[11,12]$ a model based on calculation of Jacobian of real and reactive power injection has been used for Kalman filter application. In our model, for modelling of power system dynamics formation of Jacobian matrix is not required.

The real model of power system may contain two types of errors: parametric errors and non parametric errors [13]. Parametric errors are those error whose occur in a power system due to the use of incorrect values of parameter such as incorrect value of resistance or reactance. In case of non parametric error approximate power system models are used instead of real one. In papers [14, 15], a method called extended weight least square method has been used for investing the characteristics of parametric and non parametric errors. In this paper parametric error is taken in to account and it has been observed that Kalman filter estimation is efficient for the presence of parametric error also.

The paper is organized into four sections. The introductory section provides an overview of importance of state estimator in power system operation and different methods which were briefly explained for state estimation of power system. The second section describes the modelling of power system equation for Kalman filter and WLS algorithms. WLS and Kalman filter equations along with their steps has been briefly presented in this section.

Thrid section presents the outputs of our algorithms. In this section computational efficiency, numerical stability and behaviour of our algorithm for the presence of parametric errors have been checked. Finally conclusion is provided in Section 4.

\section{Methodology}

\subsection{Modelling of power system}

The power system dynamics can be represented by the following equation:

$$
x_{k+1}=A_{k} x_{k}+w_{k}
$$

where, $x_{k}$ stands for power system state variables. $A_{k}$ is state transition matrix. $w_{k}$ is noise and nonlinearity present in the system. From the above model it is clear that system state at instant $k+1$ is dependent on the system state at instant $k$.

In our work a simple system dynamic model [9] has been used. Here it is assumed that power system is quasi static state in nature. System state variable at instant $k+1$ is same as at instant $k$ expect that state variable is subjected to small oscillations. Thus, mathematical model for the simplified system can be written as

$$
x_{k+1}=x_{k}+w_{k}
$$

where,

$$
\begin{aligned}
& x_{k}=[V \theta], \\
& V=\text { All bus voltages, } \\
& \theta=\text { All bus angles, } \\
& w_{k}=\text { Gaussian noise with zero mean. }
\end{aligned}
$$

In equation (2), system matrix $A$ is assumed as the identity matrix. This model used essentially that for linear, time invariant system [16]. For complex models $A_{k}$ will be time dependent. But for our static estimation the model presented in equation (2) is giving satisfactory results. A positive definite matrix $Q$ having zero cross correlation is assumed which is defined as the covariance matrix for our Gaussian noise $w_{k}$. The elements of the covariance matrix will depend upon power system history. Adaptive techniques can be used to tune the elements of the covariance matrix $Q$ [9]. But this is beyond the objective of this paper. Hence in this paper the value of $Q$ is assumed kept in the range $0.01-0.1$.

State estimation is a process in which states of a system is determined by a redundant set of measurements. The equation relating to state of a system measurement vector with the states can be written as

$$
z_{k}=h\left(x_{k}\right)+e
$$

where,

$$
z_{k}=\left[V, P_{i}, Q_{i}, P_{f}, Q_{f}\right],
$$

$z_{k}$ is a subset of measurement vector consisting of voltage, real power injections, reactive power injections, real power flows, reactive power flows.

$e=$ Gaussian noise in the measurement. It is assumed that noise has zero mean and its corresponding covariance matrix is denoted by $R$.

Since power system equations are nonlinear in nature, hence $h\left(x_{k}\right)$ will be a nonlinear function of state variables. In order to linearize $h\left(x_{k}\right)$ Taylor series expansion is used,

$$
z_{k}=h\left(x_{0}^{k}\right)+\frac{\delta h}{\delta h}\left(x_{k}-x_{0}^{k}\right)+v_{k}
$$

In equation (4), higher order terms are neglected and error due to linearization is included in new variable $v_{k}$. Equation (4) can be further simplified to the following form

$$
\Delta z_{k}=H_{k} \Delta x_{k}+v_{k}
$$

where, $\Delta z_{k}=z_{k}-h\left(x_{0}^{k}\right), \Delta x_{k}=x_{k}-x_{0}^{k}$ and $x_{0}^{k}$ is the initial operating point.

\subsection{WLS method}

Weight least square technique can be used for static estimation purpose. Hence, for a particular time instant equation (5) can be rewritten as

$$
\Delta z=H \Delta x+v
$$


In order to implement WLS method, numbers of measurement variable $m$ has to be more than number of state variables. Moreover the rank of Jacobian matrix should be equal to number of state variables. In WLS technique an objective function $J(x)$ which is equal to the weighted sum of squares of residuals is minimized. $J(x)$ is given by,

$$
J(x)=[z-h(x)]^{T} R^{-1}[z-h(x)]
$$

For minimization, $J(x)$ is differentiated as follows,

$$
g(x)=\frac{\delta J(x)}{\delta x}=-H^{T}(x) R^{-1}(z-h(x))=0
$$

If $g(x)$ is expanded using Taylor series expansion then,

$$
g(x)=g\left(x^{i}\right)+G\left(x^{i}\right)\left(x-x^{i}\right)
$$

where, gain matrix, $G(x)=\frac{\delta g\left(x^{i}\right)}{\delta x}$.

In the above expression higher order terms are neglected. Hence, an iterative procedure must be adopted in order to find out the estimation in which a tolerance limit has to be satisfied between successive iterations. The tolerance limit for our case is taken as $1 e-4$. The iteration will stop when the maximum value of $\Delta x$ is less tan convergence limit. The estimation of $(i+1)$ th iteration can be derived as

$$
x^{i+1}=x^{i}-\left[G\left(x^{i}\right)\right]^{-1} g\left(x^{i}\right)
$$

The computational steps used in WLS technique can be expressed as follows [1]:

1. Begin iteration, set iteration number $i=1$,

2. initialise the value of state vector,

3. read measurement inputs,

4. calculate measurement Jacobian $H(x)$,

5. calculate gain matrix $G(x)$,

6. solve for $\Delta x$,

7. check for convergence limit,

8. if required convergence criteria is not fulfilled then increment iteration index and go to Step 4.

\subsection{Kalman filter}

A Kalman filter is called as an optimal estimator. It has the capability to estimate parameters of interest from uncertain, noisy and indirect observations. Kalman filter is recursive in nature and hence it can be applied for online data estimation also. But in this paper a static estimation has been done using Kalman filter to compare the results with conventional WLS technique. The mathematical model for a Kalman filter can be expressed as follows:

$$
x_{k+1}=A x_{k}+B u_{k}+w_{k}
$$

where,

- $x_{k+1}$ and $x_{k}$ are system state variables at discrete time instant $k+1$ and $k$ respectively.

- $u_{k}$ denotes a set of control variables.

- $A$ and $B$ are matrixes which link the state variables at time $k$ to the state variables at time $k+1$.
For the above mathematical model of Kalman filter following are the necessary assumptions are taken:

$$
\begin{gathered}
E\left[x_{0}\right]=x_{0} \\
E\left[w_{k}\right]=0 \forall k \\
E\left[x_{0}, w_{k}\right]=0 \forall k \\
E\left[v_{k}\right]=0 \forall k \\
E\left[x_{0}, v_{k}\right]=0 \forall k \\
E\left[v_{j}, v_{k}\right]=0 \forall k \neq j \\
E\left[w_{j}, w\right]=0 \forall k \neq j
\end{gathered}
$$

In this paper a simplified linearised model as discussed in equations (2) and (6) are used to find out the optimal solutions. Hence it is called extended Kalaman filter algorithm. But, when iterative procedure is adopted then for each iteration the computational steps for both linear Kalman algorithm and extended Kalman algorithm will be the same.

In this algorithm it is assumed that at any time instant initial estimates, $x_{0}$ are known and its corresponding covariance matrix, $P_{K}^{0}$ is also known. Then computational steps for Kalman filter can be written as follows:

- initialise initial estimate and its associated covariance matrix,

- read a set of measurement inputs,

- calculate $\Delta z_{k}$

- calculate Jacobian matrix $H_{k}$,

- calculate Kalman gain $G_{k}$,

$$
G_{k}=P_{0} H_{k}^{T}\left(H_{k} P_{k}^{0} H_{k}^{T}+R\right)^{-1}
$$

- solve for $\Delta x$,

$$
\Delta x=G_{k}\left(\Delta z_{k}-h\left(x_{k}\right)\right)
$$

- update error covariance matrix,

$$
\begin{gathered}
P_{k}=\left(I-G_{k} H_{k}\right) P_{k}^{0} \\
P_{k+1}^{0}=P_{k}+Q_{k}
\end{gathered}
$$

- above steps are repeated for allowable tolerance limit of $\Delta x$.

Initial estimates error are considered as uncorrelated with zero mean having only diagonal entries in the covariance matrix. In this paper the initial estimates are assumed as flat i.e. bus voltage magnitudes and corresponding bus angles are 1 p.u. and $0^{\circ}$ respectively. But based on modelling of the dynamic model other conditions for initial estimates can be taken in to account [11].

\section{Results and discussion}

The effectiveness of our algorithm is checked on the IEEE 14 bus system. The single line diagram of IEEE 14 bus system is shown in Figure 1. 


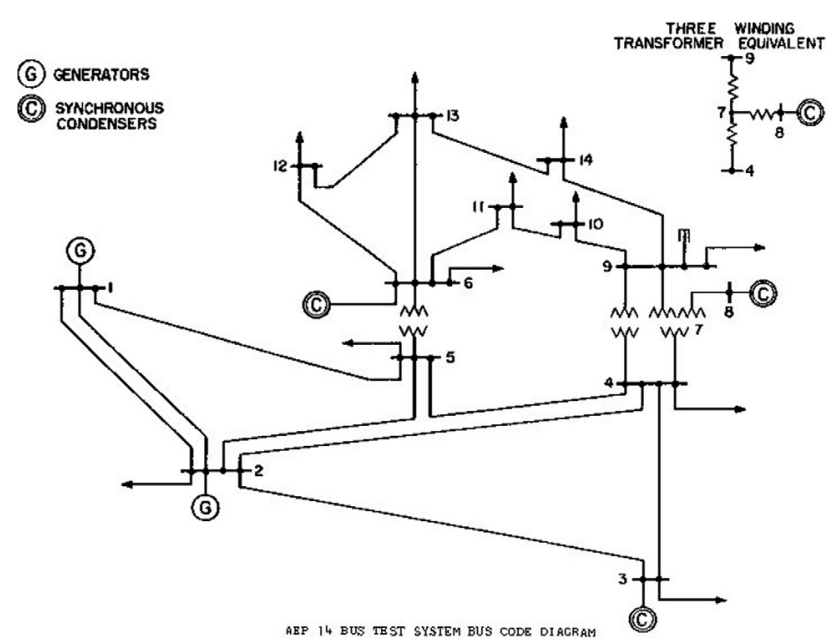

Figure 1. IEEE 14 bus test system [17].

Newton Raphson load flow study has been carried out on our test system. The results of Newton Raphson load flow analyses are shown in Tables 1 and 2.

A subset of above results consisting of bus voltage, active power injection, reactive power injection, active power flow and reactive power flow between buses are taken as the measurement vector. It is assumed that measurement vector is corrupted by zero mean Gaussian noise.

In this paper the computational efficiency of the algorithm is checked by considering parametric errors. In order to check accuracy of both conventional WLS and Kalman filter estimation, Mean Absolute Percentage Error (MAPE) [14] is used.

$$
\mathrm{MAPE}=\frac{1}{n} \sum_{i=1}^{n}\left|\frac{A_{i}-F_{i}}{A_{i}}\right| \times 100 \%
$$

where, $A_{i}$ is the actual value and $F_{i}$ is the calculated value.

A smaller value of MAPE indicates more accuracy of the estimation.

\subsection{Case 1: no parametric error}

In this case correct values of line impedances are chosen for estimation purpose.

Figure 2 shows the tracking capability of our estimated voltages. It has been found that MAPE in voltage for WLS algorithm is 5.183, but for Kalman filter estimation of voltages MAPE is 2.9961. Hence, Kalman filter estimation of voltage is more accurate than traditional WLS algorithm.

On the other hand, from Figure 3 it is observed that in the case of angle estimation Kalman filter is superior to WLS technique. MAPE value for traditional WLS is 11.3 while for Kalman estimation it is found as 2.05.

\subsection{Case 2: considering parametric error}

In this case it is assumed that bad data is present in the system. The actual value of bus impedance between bus 4 and 7 is j0.21 ohm but here it is taken as j0.4 ohm.
Table 1. Real and reactive power flow.

\begin{tabular}{cccc}
\hline From bus & To bus & \multicolumn{2}{c}{ Power flow (p.u) } \\
\cline { 3 - 4 } & & Real & Reactive \\
\hline 1 & 2 & 1.571 & -0.175 \\
1 & 5 & 0.755 & -0.0798 \\
2 & 3 & 0.734 & 0.059 \\
2 & 4 & 0.559 & 0.029 \\
2 & 5 & 0.417 & 0.0473 \\
3 & 4 & -0.231 & 0.077 \\
4 & 5 & -0.595 & 0.116 \\
4 & 7 & 0.271 & -0.154 \\
\hline
\end{tabular}

Table 2. Real and reactive power flow.

\begin{tabular}{cccrr}
\hline Bus no & Voltage (p.u) & Angle (degree) & \multicolumn{2}{c}{$\begin{array}{r}\text { Power injection } \\
\text { (p.u) }\end{array}$} \\
\cline { 3 - 5 } & & & Real & Reactive \\
\hline 1 & 1.060 & 0 & 2.326 & -0.152 \\
2 & 1.045 & -4.9891 & 0.183 & 0.352 \\
3 & 1.010 & -12.7492 & -0.942 & 0.088 \\
4 & 1.0132 & -10.2420 & -0.478 & 0.039 \\
5 & 1.0166 & -8.7601 & -0.076 & -0.016 \\
6 & 1.0700 & -14.4469 & -0.112 & 0.155 \\
7 & 1.0457 & -13.2368 & -0.000 & 0.000 \\
8 & 1.0800 & -13.2368 & 0.000 & 0.210 \\
9 & 1.0305 & -14.8201 & -0.295 & -0.166 \\
10 & 1.0299 & -15.0360 & -0.090 & -0.058 \\
11 & 1.0461 & -14.8581 & -0.035 & -0.018 \\
12 & 1.0533 & -15.2937 & -0.061 & -0.016 \\
13 & 1.0466 & -15.3313 & -0.135 & -0.058 \\
14 & 1.0193 & -16.0717 & -0.149 & -0.050 \\
\hline
\end{tabular}

The comparision of estimation for voltage and angles are shown in Figures 4 and 5 respectively.

It has been found that WLS and Kalman estimation MAPE values for voltage estimation are 4.38 and 3.48 respectively. On the other hand, MAPE values for angle estimations using WLS and Kalman techniques are 15.22 and 4.2876 respectively. Since Kalman estimation has less value of MAPE, hence it is more accurate than WLS estimation.

\subsection{Numerical stability of Kalman algorithm}

In this paper initial estimate is assumed as a flat start in the Kalman filter estimation algorithm. The error covariance in the initial estimates is taken as 0.01 . In order to show the stability of this algorithm error covariance is increased to $50 \%$ of its initial value. The estimation results of voltage and angles for different set of values of error covariances are shown in Tables 3 and 4 respectively. It is observed that for both voltage and angle estimation the values are approximately matching each other.

\subsection{Convergence analysis}

In order to check the convergence ability of Kalman algorithm bus 2 voltage is chosen. The initial estimate of bus 2 voltage is 1 p.u. and its actual value is 1.05 p.u. 


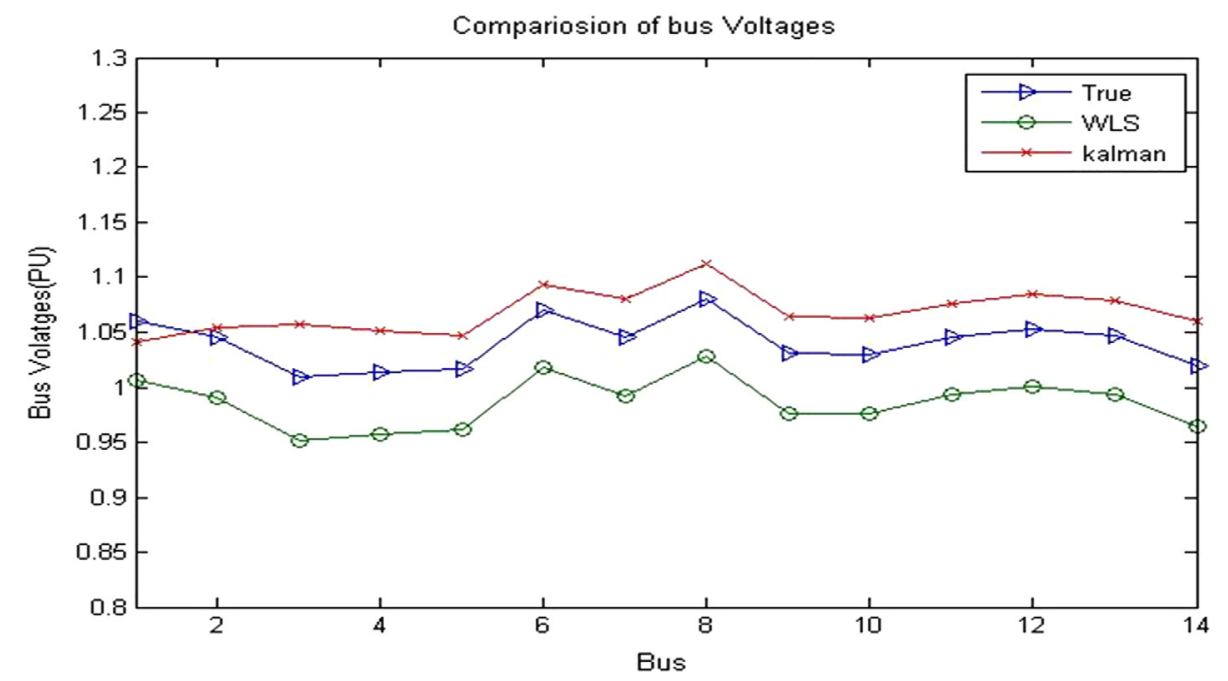

Figure 2. Comparision of estimated bus voltages.

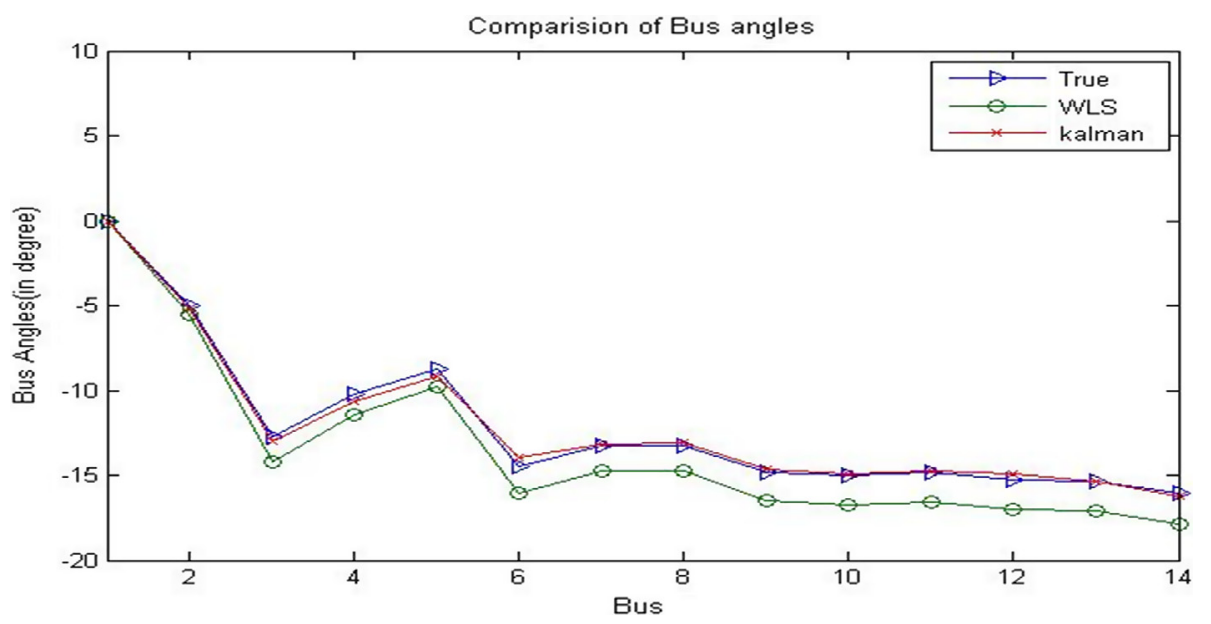

Figure 3. Comparision of estimated bus angles.

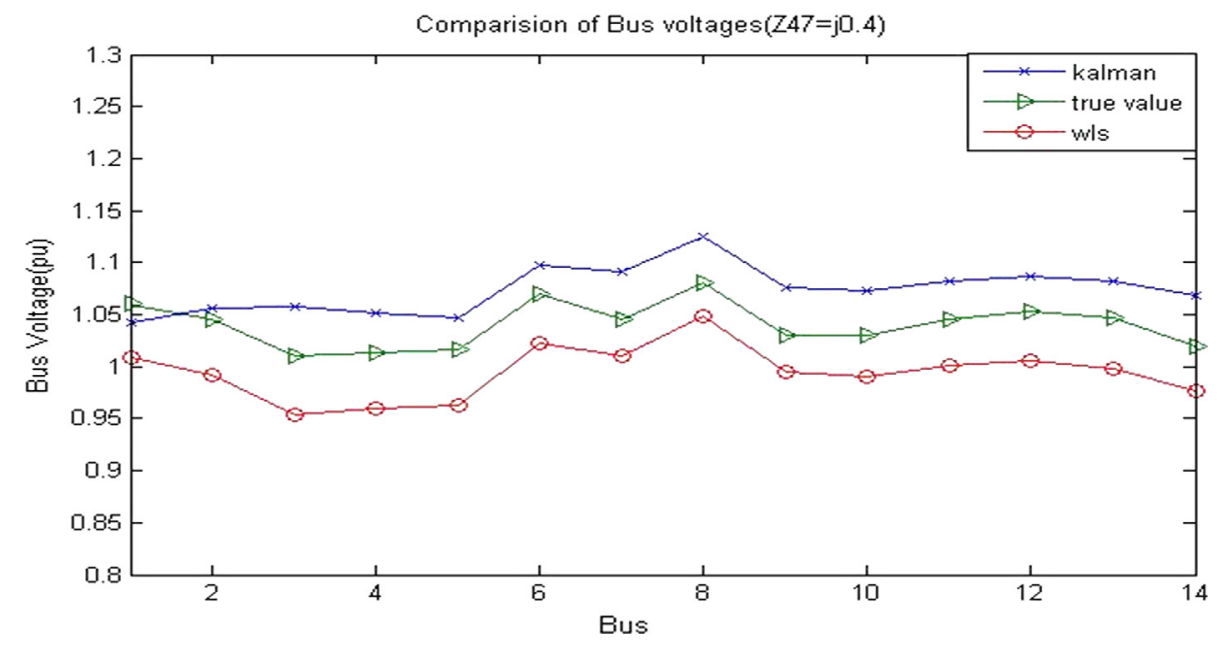

Figure 4. Comparision of bus voltages, $Z_{47}=\mathrm{j} 0.4 \mathrm{ohm}$. 


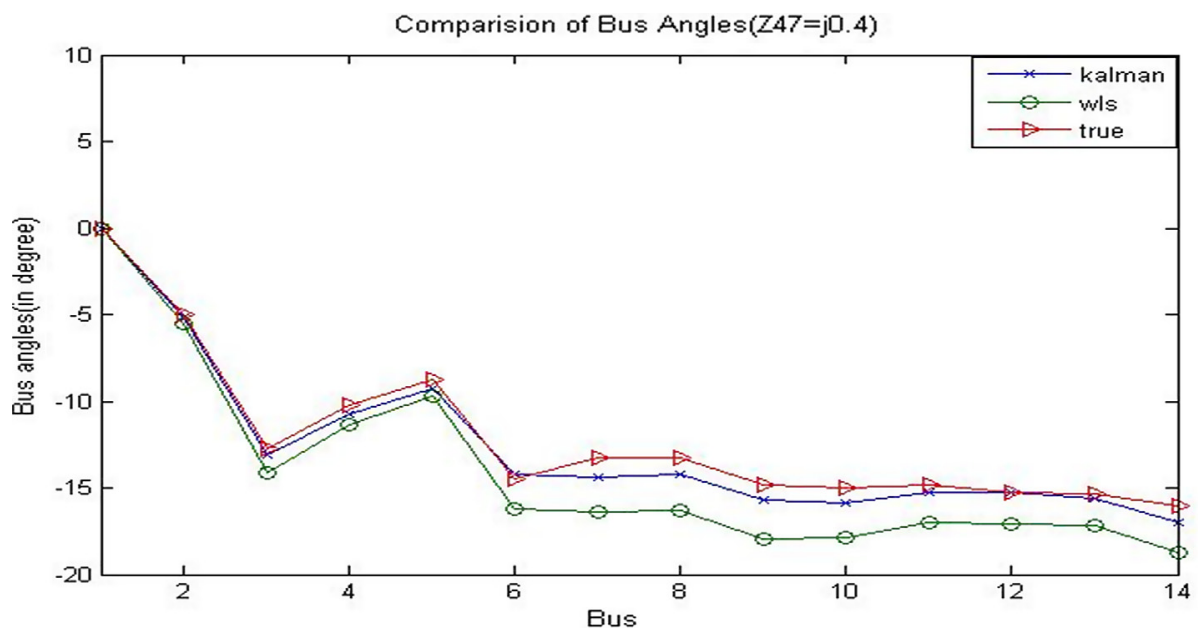

Figure 5. Comparision of bus angles, $Z_{47}=\mathrm{j} 0.4 \mathrm{ohm}$.

Table 3. Kalman filter voltage estimation for different values of initial error covariance matrix.

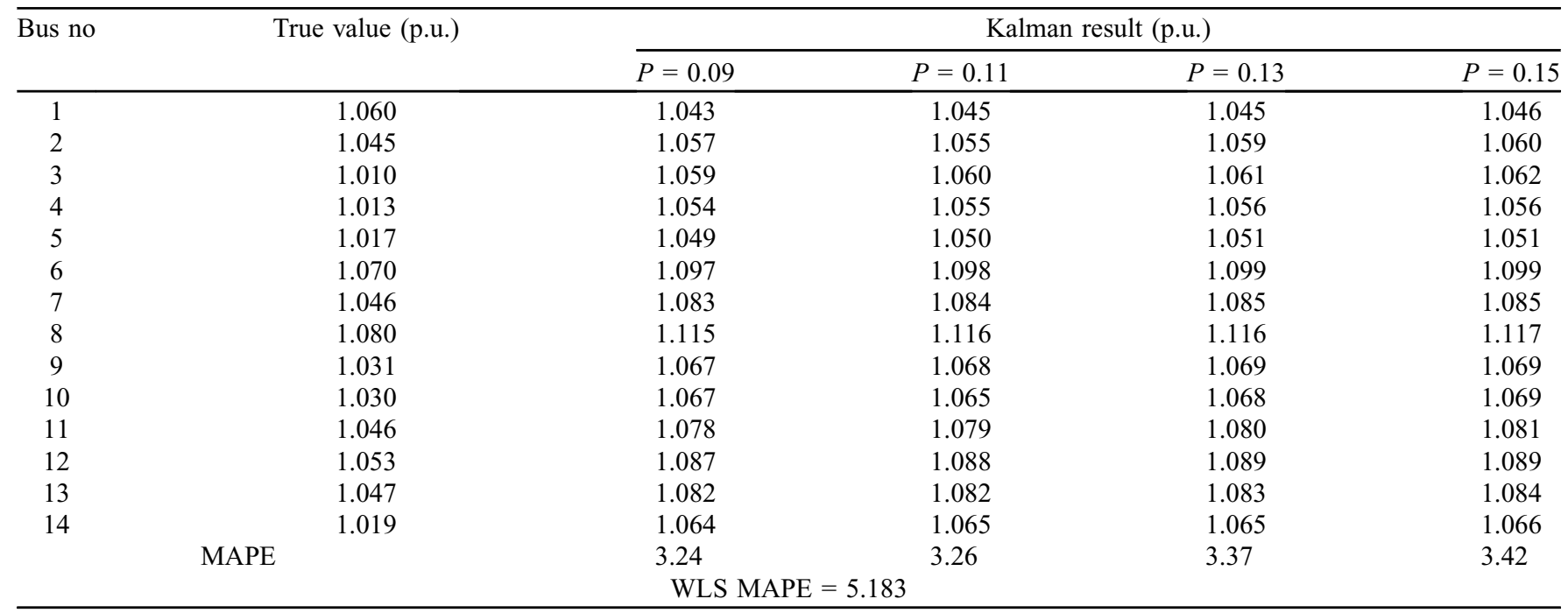

Table 4. Kalman filter angle estimation for different values of initial error covariance matrix.

\begin{tabular}{|c|c|c|c|c|c|}
\hline \multirow[t]{2}{*}{$\overline{\text { Bus no }}$} & \multirow[t]{2}{*}{ True value (degree) } & \multicolumn{4}{|c|}{ Kalman result (degree) } \\
\hline & & $P=0.09$ & $P=0.11$ & $P=0.13$ & $P=0.15$ \\
\hline 1 & 0.00 & 0 & 0.00 & 0.00 & 0.00 \\
\hline 2 & -4.99 & -5.16 & -5.16 & -5.15 & -5.15 \\
\hline 3 & -12.75 & -12.96 & -12.95 & -12.93 & -12.92 \\
\hline 4 & -10.25 & -10.61 & -10.60 & -10.59 & -10.59 \\
\hline 5 & -8.76 & -9.16 & -9.15 & -9.15 & -9.15 \\
\hline 6 & -14.45 & -13.90 & -13.90 & -13.90 & -13.90 \\
\hline 7 & -13.24 & -13.12 & -13.11 & -13.11 & -13.11 \\
\hline 8 & -13.24 & -13.01 & -13.00 & -12.99 & -12.99 \\
\hline 9 & -14.83 & -14.55 & -14.53 & -14.53 & -14.53 \\
\hline 10 & -15.04 & -14.89 & -14.87 & -14.87 & -14.87 \\
\hline 11 & -14.86 & -14.65 & -14.64 & -14.63 & -14.64 \\
\hline 12 & -15.31 & -14.90 & -14.90 & -14.90 & -14.90 \\
\hline 13 & -15.34 & -15.25 & -15.24 & -15.24 & -15.25 \\
\hline 14 & -16.08 & -16.17 & -16.16 & -16.15 & -16.16 \\
\hline \multirow{2}{*}{\multicolumn{2}{|c|}{ MAPE }} & 2.12 & 2.13 & 2.11 & 2.09 \\
\hline & & WLS N & & & \\
\hline
\end{tabular}




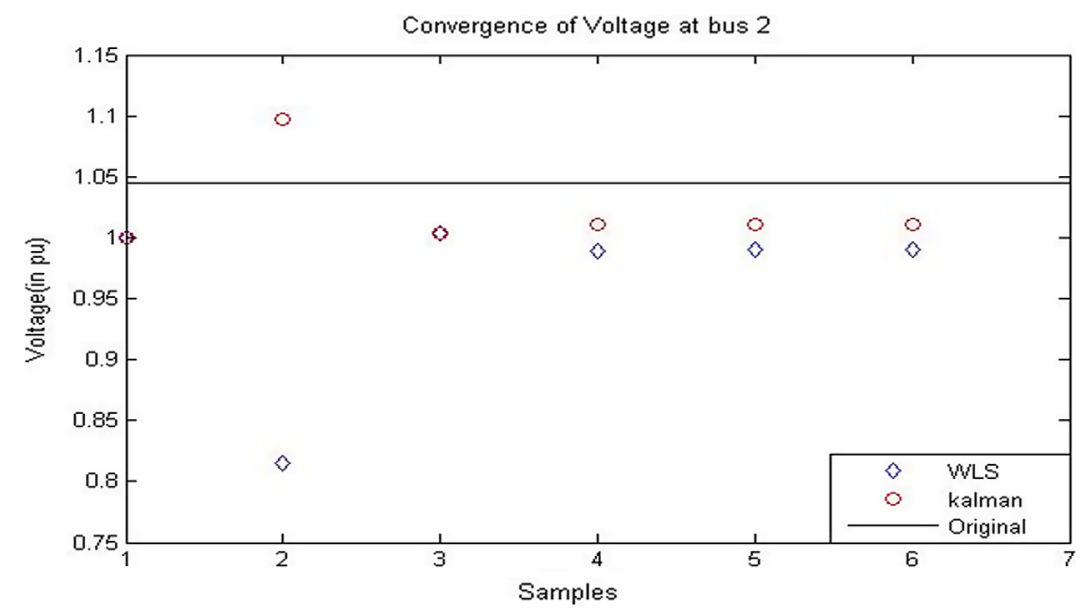

Figure 6. Convergence of voltage at bus 2 .

Figure 6 shows the estimated values of voltage at bus 2 for successive samples. It has been observed that for successive samples the error in Kalman estimation is lesser than that of traditional WLS technique.

\section{Conclusion}

In this paper a comparative study has been done between WLS technique and Kalman filter algorithm for static state estimation of power system. Simple power system dynamic equation and measurement model are considered in order to implement this algorithm. Algorithms are tested on IEEE 14 bus system and it is found that Kalman filter has better estimation quality than WLS algorithm. The results presented in this paper prove that for the presence of parametric error also Kalman estimation outperform traditional WLS estimation.

From the results it can be concluded that in the face of errors in initial estimates, Kalman algorithm is numerically stable. The benefit of Kalman estimation is that this method has the ability to estimate more accurately than conventional WLS technique and it has better convergence characteristics.

\section{References}

1. Abur A, Exposito AG. 2004. Power system state estimation, theory and implementation. Marcel Dekker Inc.: New York.

2. Meliopoulos APS, Fardanesh B, Zelingher S. 2001. Power system state estimation: modeling error effects and impact on system operation. Proceedings of the Hawaii International Conference on System Sciences, January 3-6, 2001, Maui, Hawaii.

3. Schweppe F, Wildes J. 1970. Power system static-state estimation, part 3: implementation. IEEE Power Apparatus and Systems, 89, 130-135.

4. Holten L, Wu FF, Liu W-HE. 1988. Comparison of different methods for state estimation. IEEE Transactions on Power Systems, 3(4), 1798-1806.
6. Grainger J, Stevenson W. 1994. Power system analysis. McGraw-Hill: New York.

5. Kalman RE. 1960. A new approach to linear filtering and prediction problems. Transactions of the ASME-Journal of Basic Engineering, 82(Series D), 35-45.

7. Girgis AA. 1982. A new Kalman filtering based digital distance relay. IEEE Transactions on Power Apparatus and System, PAS-101(9), 3471-3480.

8. Sachdev MS, Wood HC, Johnson NG. 1985. Kalman filtering applied to power system measurements for relaying. IEEE Transactions on Power Apparatus and System, PAS-104(12), 4565-4573.

9. Beides HM, Heydt GT. 1991. Dynamic state estimation of power system harmonics using Kalman filter methodology. IEEE Transactions on Power Delivery, 6(4), 1663-1670.

10. Julier SJ, Uhlmann JK. 1997. A new extension of the Kalman filter to nonlinear systems. Proc. SPIE 3068 Signal Processing Sensor Fusion, and Target Recognition VI, Florida, 28 July, 1997.

11. Blood EA, Krogh BH, Ilic MD. 2008. Electric power system static state estimation through Kalman filtering and load forecasting. Power and Energy Society General Meeting Conversion and Delivery of Electrical Energy in the 21st Century, Pittsburg, USA, 2008 IEEE.

12. Hernandez C, Maya-Ortiz P. 2015. Comparison between WLS and Kalman filter method for power system static state estimation. Smart Electric Distribution Systems and Technologies (EDST) 2015 International Symposium on, Vienna, Austria.

13. Zarco P, Exposito AG. 2000. Power system parameter estimation: a survey. IEEE Transactions on Power Systems, 15(1), 216-222.

14. Chen J, Lio Y. 2012. State estimation and power flow analysis of power system. Journal of Computers, 7(3), 685-691.

15. Liao Y. 2007. State estimation algorithm considering effects of model inaccuracies. IEEE Proceedings, SoutheastCon 2007, Richmond, Virginia.

16. Debs AS, Larson RE. 1970. A dynamic estimator for tracking the state of power system. IEEE Transactions on Power Apparatus and System, PAS-89(7), 1670-1678.

17. Power System Test Case Archive, http://www2.ee.wasington.edu/ researc/pstca/. 